\title{
Cystine Dimethylester Model of Cystinosis: Still Reliable?
}

\author{
MARTIJN J. WILMER, PETER H. WILLEMS, SJOERD VERKAART, HENK-JAN VISCH, ADRIANA DE GRAAF-HESS, \\ HENK J. BLOM, LEO A. MONNENS, LAMBERTUS P. VAN DEN HEUVEL, AND ELENA N. LEVTCHENKO
}

\begin{abstract}
Laboratory of Pediatrics and Neurology [M.J.W., A.G.-H., H.J.B., L.P.H.], Radboud University Nijmegen Medical Centre, 6500 HB, Nijmegen, The Netherlands; Department of Biochemistry [P.H.W., S.V., H.-J.V.], Nijmegen Centre for Molecular Life Sciences and Nijmegen Centre for Mitochondrial Disorders, Radboud University Nijmegen Medical Centre, 6500 HB, Nijmegen, The Netherlands; Department of Pediatric Nephrology [L.A.M., L.P.H., E.N.L], Radboud University Nijmegen Medical Centre, 6500 HB, Nijmegen, The Netherlands
\end{abstract}

\begin{abstract}
The ability of cystine dimethylester (CDME) to load lysosomes with cystine has been used to establish the basic defect in cystinosis: defective cystine exodus from lysosomes. Using CDME loading, it has been postulated that cystine accumulation in cystinosis affects mitochondrial ATP production, resulting in defective renal tubular reabsorption. Recent studies in cystinotic fibroblasts, however, show normal adenosine triphosphate (ATP) generation capacity. To investigate the effect of CDME in more detail, mitochondrial ATP generation, reactive oxygen species production, and viability are compared in fibroblasts loaded with CDME with those of cystinotic cells with a defective cystine transporter. Intracellular cystine levels were comparable in fibroblasts loaded with CDME (1 mM, 30 min) and cystinotic fibroblasts. Intracellular ATP levels and mitochondrial ATP production were decreased in fibroblasts loaded with CDME, but normal in cystinotic fibroblasts. Superoxide production was increased with $300 \%$ after CDME loading, whereas no changes were observed in cystinotic fibroblasts. Exposure to CDME led to cell death in a time- and concentration-dependent manner. Our data demonstrate that CDME has a toxic effect on mitochondrial ATP production and cell viability. These effects are not observed in cystinotic cells, indicating that a more appropriate model is required for studying the pathogenesis of cystinosis.
\end{abstract}

(Pediatr Res 62: 151-155, 2007)

L ysosomal cystine accumulation is the hallmark of the lysosomal storage disorder cystinosis, caused by a defect in the lysosomal cystine carrier cystinosin encoded by the CTNS gene (1). Cystinosis is the most common cause of inborn Fanconi syndrome, which generally progresses toward end-stage renal disease during the first decade of life when untreated with the cystine-depleting agent cysteamine (2). Defective cystine exodus from cystinotic lysosomes was established as the basic defect in cystinosis (3) by using a technique of lysosomal loading with CDME developed by Goldman and Kaplan (4) and Reeves (5). Subsequently, proximal tubular cells (PTEC) loaded with CDME were used for studying the pathogenesis of cystinosis, because no in vivo or in vitro model of cystinosis carrying a defect in the CTNS gene was available at that time.

\footnotetext{
Received November 21, 2006; accepted March 9, 2007.

Correspondence: Martijn J. Wilmer, M.D., Laboratory of Pediatrics and Neurology (656), Radboud University Nijmegen Medical Centre, P.O. 9101, 6500 HB Nijmegen, The Netherlands; e-mail: m.wilmer@cukz.umcn.nl.

This study was supported by the Dutch Kidney Foundation (PC 106).
}

CDME loading of rat and rabbit isolated proximal tubules and of a porcine epithelial tubular cell line (LLC-PK1) caused defective proximal tubular reabsorption, comparable with Fanconi syndrome in cystinosis (6-8). Using this model, it was postulated that altered mitochondrial ATP generation could be the underlying pathogenic mechanism of cystinosis because CDME loading resulted in a dramatic decrease of mitochondrial ATP production, leading to decreased activity of proximal tubular Na,K-ATPase (9-11). As the majority of proximal tubular transporters are $\mathrm{Na}^{+}$-coupled, decreased activity of $\mathrm{Na}, \mathrm{K}-\mathrm{ATPase}$, leading to a decreased transmembrane $\mathrm{Na}^{+}$gradient, was suggested to cause generalized dysfunction of proximal tubular transport in cystinosis (12). This hypothesis established in CDME-loading model has recently been challenged by in vitro studies in cells derived from cystinotic patients.

Our group extensively studied energy metabolism in cultured cystinotic fibroblasts. ATP generation in these cells appeared to be normal under basal and stimulated conditions. A moderate decrease in intracellular ATP content did not cause alterations of Na,K-ATPase activity (13). Furthermore, we found normal intracellular ATP levels in human immortalized PTEC, derived from urine of cystinotic patients compared with healthy controls (14). Similar to our results, normal intracellular ATP content was demonstrated in primary cystinotic PTEC under basal conditions by Laube et al. (15).

Because the results of the studies mentioned above are in disagreement with those obtained in PTEC exposed to CDME, we assumed that CDME loading could provoke effects different from those attributed to lysosomal cystine accumulation in cells with a deficient lysosomal cystine carrier. In concordance with this idea, a recent study using the IHKE-1 cell line demonstrated that CDME had an acute effect on the basal membrane potential, possibly due to activated $\mathrm{K}^{+}$conductance (16). In the present study, we investigated the effect of CDME loading on mitochondrial ATP-generating capacity, superoxide production, and viability of control fibroblasts compared with cystinotic fibroblasts accumulating cystine due to a known genetic defect of the CTNS gene.

Abbreviations: CDME, cystine dimethylester; HEt, hydroethidine; PTEC, proximal tubular epithelial cells 


\section{METHODS}

Cell cultures. Fibroblasts were cultured from skin biopsies after obtaining informed consent of healthy controls (ct1 and ct2) and cystinotic patients (pt1 and pt2), as described previously (13). Cystinosis was diagnosed in all patients by measuring elevated cystine content in polymorphonuclear cells ( $>0.5 \mathrm{nmol}$ cystine $/ \mathrm{mg}$ protein) and was confirmed by molecular analysis of the CTNS gene. Both patients carried the common homozygous 57-kb deletion of the CTNS gene.

Cystine loading and determination of intracellular cystine content. Control cells were loaded by adding CDME (Fluka) to culture medium at a final concentration of $1.0 \mathrm{mM}$ and incubated for $30 \mathrm{~min}$ at $37^{\circ} \mathrm{C}$ and $5 \% \mathrm{CO}_{2}$ before further analysis. Pilot experiments showed that cystine concentrations under these conditions were in the range observed in cystinotic fibroblasts. For concentration-dependent assays, CDME concentration ranged from 0.1 to $5.0 \mathrm{mM}$ and for the time-dependent assays, CDME incubation time varied from 10 to $120 \mathrm{~min}$. After CDME loading, cells were rinsed briefly with phosphate-buffered saline (PBS) before harvesting using trypsinethylenediamine tetraacetic acid (EDTA). After detachment, cells were further processed on ice to prevent cystine exodus from lysosomes. Intracellular cystine levels were determined as described previously and expressed as nmol cystine/mg protein (equal to ( $1 / 2$ cysteine/mg protein)/2) (17).

Intracellular ATP content and mitochondrial ATP production. After culturing fibroblasts to confluence, cells were incubated with CDME as indicated above (1.0 $\mathrm{mM} \mathrm{CDME}$ for $30 \mathrm{~min}$ ) and harvested using trypsinEDTA to determine intracellular ATP levels in three independent experiments using an ATP luminescence kit (Roche) as described previously. Data are presented as nmol ATP/mg protein (13) and expressed as mean \pm SEM.

Mitochondrial ATP production was monitored by transfecting fibroblasts cultured on a coverslip with a baculovirus containing cDNA for mitochondria-targeted luciferase $(13,18)$. Perfusing the transfected cells with $\mathrm{N}$-2hydroxyethylpiperazine- $N{ }^{1}$-2-ethanesulfonic acid (HEPES)-Tris medium (132 mM NaCl, $4.2 \mathrm{mM} \mathrm{KCl}, 1 \mathrm{mM} \mathrm{MgCl}$, $5.5 \mathrm{mM}$ D-glucose, $10 \mathrm{mM}$ HEPES, $1 \mathrm{mM} \mathrm{CaCl}_{2}, \mathrm{pH}$ 7.4) supplemented with $5 \mu \mathrm{M}$ beetle luciferin allowed us to monitor mitochondrial ATP production in intact cells at $37^{\circ} \mathrm{C}$ using a photomultiplier tube. After stabilization of the signal, $1 \mathrm{mM} \mathrm{CDME}$ was added to the perfusion medium at the indicated time. To investigate whether the effect of CDME on mitochondrial ATP production in the cells was reversible, CDME was removed after $15 \mathrm{~min}$ from the perfusion medium. Results were expressed as a relative decrease in luciferase luminescence. Concentration-dependent effects of CDME perfusion $(0.2,0.5,1.0$, and 5.0 $\mathrm{mM}$ ) on mitochondrial ATP production were determined in one control fibroblast cell line. The luminescence was normalized to the initial basal level for each concentration and set to $100 \%$. The relative luminescence for all CDME concentrations was plotted against time.

Measurement of Na,K-ATPase activity using ${ }^{86} \mathrm{Rb}^{+}$. The uptake of ${ }^{86} \mathrm{Rb}^{+}$, a congener of $\mathrm{K}^{+}$, can be used to determine the $\mathrm{Na}, \mathrm{K}$-ATPase activity in a monolayer of fibroblasts. To study the effect of CDME loading on $\mathrm{Na}, \mathrm{K}-\mathrm{ATP}$ ase activity, we measured ${ }^{86} \mathrm{Rb}^{+}$uptake with or without inhibitor ouabain $(10 \mathrm{mM})$, as described previously (13). Uptake of ${ }^{86} \mathrm{Rb}^{+}$has been evaluated in two control fibroblasts with or without 30-min loading with CDME (1 mM) and compared with cystinotic fibroblasts.

Quantification of mitochondrial superoxide levels. Control and cystinotic fibroblasts were cultured on coverslips and incubated in HEPES-Tris medium containing $10 \mu \mathrm{M}$ hydroethidine (HEt) (Molecular Probes, Leiden, The Netherlands) for $10 \mathrm{~min}$ at $37^{\circ} \mathrm{C}$. HEt is a lipophilic, uncharged compound that enters the cell and reacts with superoxide to form two positively charged products, 2-hydroxyethidium and ethidium, both of which emit red fluorescence (19). The reaction was stopped by thoroughly washing the cells with PBS to remove excess HEt. For quantitative analysis of the fluorescent HEt oxidation products, coverslips were mounted in an incubation chamber placed on the stage of an inverted microscope (Axiovert 200 M, Carl Zeiss, Jena, Germany) equipped with a Zeiss $40 \times / 1.3$ NA fluor objective. The cells were excited at $490 \mathrm{~nm}$ using a monochromator (Polychrome IV, TILL Photonics, Gräfelfing, Germany). Fluorescence emission light was directed by a 525DRLP dichroic mirror (Omega Optical Inc., Brattleboro, VT) through a 565ALP emission filter (Omega) onto a CoolSNAP HQ monochrome CCDcamera (Roper Scientific, Vianen, The Netherlands). Hardware was controlled with Metafluor 6.0 software (Universal Imaging Corporation, Downingtown, PA). Subsequently, control cells $(n=2)$ were loaded for $2 \mathrm{~h}$ with $0.5 \mathrm{mM}$ CDME and monitored again. Routinely, 10 fields of view with two to five cells each were analyzed per coverslip using an acquisition time of $100 \mathrm{~ms}$. Data are presented as the percentage of increase of control fibroblast ct1 \pm SEM.

Viability assay. The viability of cultured fibroblasts and human PTEC line HK-2 after treatment with CDME was tested in 96 wells by a fluorescent assay using resazurin $(0.01 \%)$ as a marker (20). Subsequent to CDME incubations at various concentrations and time periods, culture medium was aspirated and cells were incubated with resazurin for $2.5 \mathrm{~h}$, before measuring fluorescence at $590 \mathrm{~nm}$ with excitation of $560 \mathrm{~nm}$. Viability was expressed as the percentage of fluorescence compared with vehicle control. Additionally, viability was tested using trypan blue exclusion.

Statistical analysis. Wilcoxon signed rank test was used for comparing intracellular ATP levels and ${ }^{86} \mathrm{Rb}^{+}$uptake. A $t$ test was used for comparing mitochondrial superoxide levels between control and cystinotic fibroblasts at basic conditions and control fibroblasts after CDME loading. The differences were considered statistically significant at $p<0.05$.

\section{RESULTS}

Cystine accumulation in loaded control fibroblasts and unloaded cystinotic fibroblasts. The two cystinotic fibroblasts pt1 and pt2 accumulated cystine (1.83 and 5.14 nmol cystine/mg protein, respectively) compared with two untreated controls (0.09 and $0.09 \mathrm{nmol}$ cystine/mg protein, respectively), consistent with defective lysosomal cystine transporter in cystinotic cells. Loading of control fibroblasts with $1 \mathrm{mM}$ CDME for $30 \mathrm{~min}$ resulted in an increase in intracellular cystine levels (10.36 and $3.41 \mathrm{nmol}$ cystine/mg protein in control ct1 and ct2, respectively). In preliminary CDMEloading experiments in the two control fibroblasts, we found that loading for $10 \mathrm{~min}$ resulted in lower cystine levels: 0.70 and $0.55 \mathrm{nmol}$ cystine/mg protein, respectively.

Effect of CDME on intracellular ATP content and mitochondrial ATP production. In three independent experiments, intracellular ATP levels in control fibroblasts loaded with CDME were significantly decreased $(p<0.05)$ after 30 min of incubation with $1 \mathrm{mM}$ CDME, whereas cystinotic cells displayed ATP levels in the range of untreated controls (Fig. $1 A)$. Monitoring luminescence in loaded control fibroblasts transfected with mitochondria-targeted luciferase demonstrated an acute decrease in mitochondrial ATP levels when cells were perfused with $1 \mathrm{mM}$ CDME in addition to luciferin (Fig. 1B). Within $10 \mathrm{~min}$, mitochondrial ATP production decreased to background levels. The effect of CDME on mitochondrial ATP production appeared to be dependent on its concentration (Fig. 1B). When loading cystinotic fibroblasts, the effects on mitochondrial ATP production were similar to control fibroblasts: an acute decrease in mitochondrial ATP production during CDME perfusion (data not shown). Furthermore, removal of CDME from the perfusion medium 15 min after the exposure did not result in restoration of ATP production during the next $40 \mathrm{~min}$ (data not shown).

Effect of CDME on Na,K-ATPase activity. The effect of 30-min CDME loading in control fibroblasts on ${ }^{86} R b^{+}$uptake was not significantly altered. This effect was similar in cystinotic fibroblasts compared with control cells.

Mitochondrial superoxide levels. Using the fluorescent products of HEt oxidation, we could measure the superoxide levels before and after CDME loading of control fibroblasts. CDME loading resulted in a significant increase in superoxide levels in control fibroblasts $(300 \%$ increase, $p<0.001)$, whereas no significant difference could be detected between cystinotic and unloaded control cells (Fig. 2).

Viability assay. Incubating control fibroblasts for 10-120 min with $0.1-3 \mathrm{mM}$ of CDME clearly showed a gradual decrease in fluorescence of resazurin, indicating that viability of the cells was reduced by CDME (Fig. 3A). In the fibro- 
A

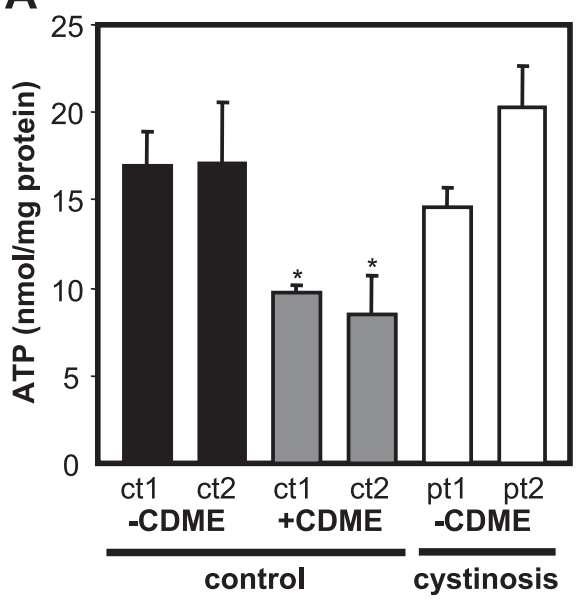

B

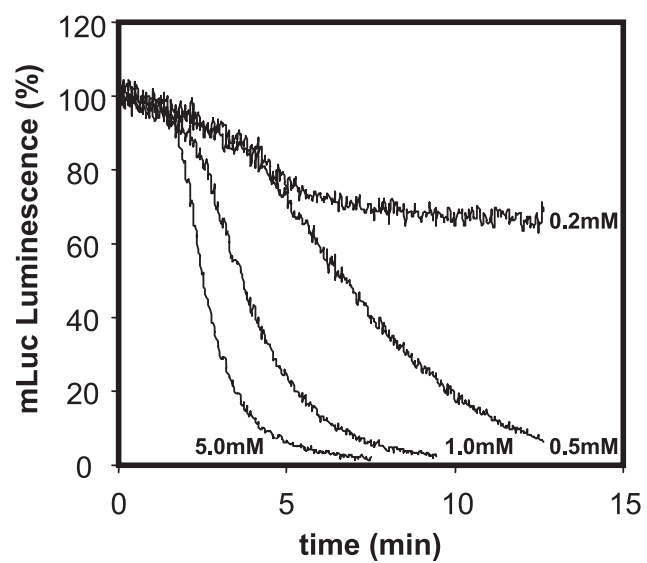

Figure 1. CDME loading results in decreased ATP levels and mitochondrial ATP production. (A) Intracellular ATP levels were measured using an ATP luminescence kit from Roche in two control cell lines (ct1 and ct2) without and with CDME (1 mM, $30 \mathrm{~min})$ and compared with ATP levels of two cell lines derived from cystinotic patients (pt1 and pt2). (B) Monitoring mLuc luminescence representing mitochondrial ATP production in control fibroblast ct1 transfected with mitochondria-targeted luciferase and perfused with various concentrations of CDME. Plotted as the percentage of luminescence compared with basal levels before CDME perfusion. Data are presented as mean nmol ATP/mg protein \pm SEM. *Significantly different from control fibroblast ct1 $(p<0.05)$.

blasts, 30 min-incubation with $0.5 \mathrm{mM}$ CDME resulted in a viability of at least $85 \%$, whereas incubations as long as 120 min and with as much as $3 \mathrm{mM}$ killed more than $90 \%$ of the cells. Determination of viability using trypan blue exclusion after incubation with $1 \mathrm{mM} \mathrm{CDME}$ at the same time intervals showed similar results (data not shown).

Because PTEC might be more resistant to CDME toxicity, we performed the similar experiments in the human HK-2 PTEC line. The results obtained were comparable with those obtained in cultured fibroblasts (Fig. 3B).

\section{DISCUSSION}

CDME loading of PTEC has been extensively used for studying the pathogenesis of cystinosis. Using this model, the hypothesis of altered ATP metabolism being the keystone in the pathogenesis of cystinosis has been postulated $(9-11)$. In

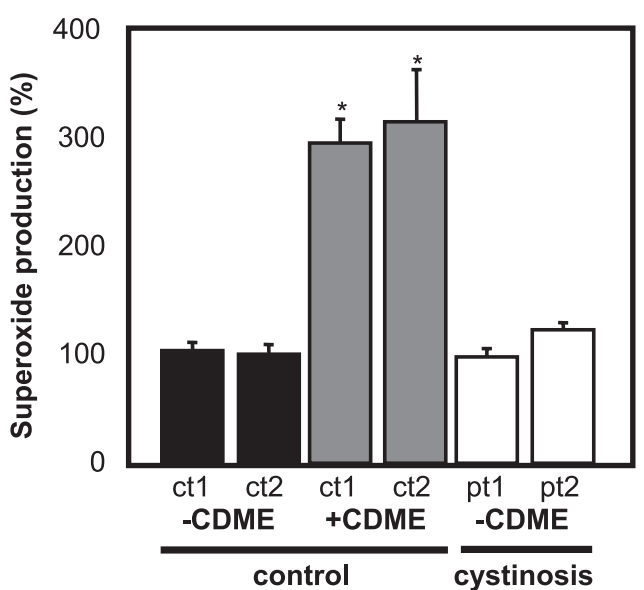

Figure 2. Superoxide production is increased after CDME loading. Superoxide production in two control fibroblasts loaded with or without CDME loading $(2 \mathrm{mM}, 2 \mathrm{~h})$ and two cystinotic fibroblasts. Relative superoxide production is determined by measuring fluorescence at $565 \mathrm{~nm}$ and excitation at $490 \mathrm{~nm}$ after HEt incubation and compared with control fibroblast ct1, without CDME loading. Data are presented as mean percentage of increase of control fibroblast ct1 ( \pm SEM). *Significantly different from control fibroblast ct1 $(p<0.001)$.
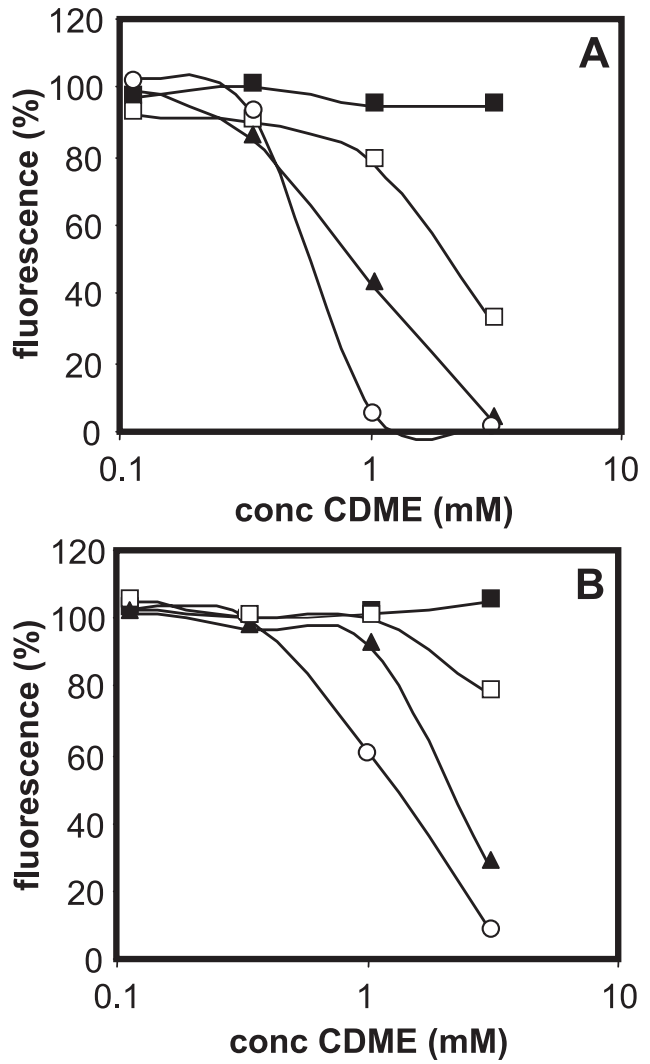

Figure 3. Viability of cultured cells is decreased due to CDME loading. Concentration- and time-dependent viability after CDME loading $(1 \mathrm{mM})$ in control fibroblasts $(A)$ and control HK-2 $(B)$. Viability is determined using resazurin by measuring fluorescence at $590 \mathrm{~nm}$ with excitation at $560 \mathrm{~nm}$ and expressed as the percentage of fluorescence compared with vehicle control. Viability is measured after $10 \min (\mathbf{\square}), 30 \mathrm{~min}(\square), 60 \mathrm{~min}(\mathbf{\Delta})$, and $120 \min (\mathrm{O})$.

this study, we demonstrated that CDME loading immediately reduces mitochondrial ATP generation and additionally causes extensive superoxide production and cell death of the 
loaded cells. These results cannot be attributed to lysosomal cystine accumulation because cystinotic fibroblasts displaying comparable intracellular cystine content, demonstrated normal mitochondrial ATP levels, unchanged superoxide production, and preserved viability compared with unloaded control cells. Furthermore, a threefold difference in cystine concentration between the two control fibroblasts cultures after CDME loading was not associated with remarkable differences in ATP levels in the present study. This is in concordance with our previous study, demonstrating no correlation between cystine levels and ATP levels (13).

In this study, we did not explore the exact mechanism of CDME toxicity. However, we might speculate that the toxic effect of CDME could be caused by a high oxidative potential of this ester, which is hydrolyzed in the lysosomes to cystine and methanol and subsequently exported from the lysosomes via intact cystinosin in these cells. Toxic effects of methanol, released after CDME hydrolysis within the cells, probably does not play a major role because the exposure of cells to the equal quantities of leucine, tryptophan, and alanine ester did not produce any measurable toxic effect $(6,8)$. Loading cells with millimolar amounts of CDME, as it has been done in the majority of studies using this ester, will thus deliver millimoles of cystine to the cytosol. These high cystine concentrations can either react with free sulfhydryl (-SH) groups of proteins and polypeptides via a disulfide exchange reaction, disturbing their function, or can be reduced to cysteine. The latter will consume a substantial amount of intracellular antioxidants (e.g. glutathione) and make cells prone to oxidative damage.

In our study, the effect of CDME on the mitochondrial ATP generation was observed within the first minute after the exposure, suggesting a toxic effect on the mitochondria. Remarkably, the inhibitory effect of CDME on the mitochondrial ATP generation was irreversible, probably due to the permanent oxidative damage of the mitochondrial membrane produced by this ester. Interestingly, a direct effect of CDME on isolated mitochondria has already been demonstrated by Foreman et al. (11), who observed a significant decline of mitochondrial $\mathrm{O}_{2}$ utilization after CDME exposure using glutamate as a substrate, but not with succinate, pointing to the initial inhibition of the respiratory complex I.

Interestingly, in contrast to earlier studies, we found normal $\mathrm{Na}, \mathrm{K}-\mathrm{ATPase}$ activity after CDME loading $(6,9)$. The explanation of this observation could be that the $50 \%$ decrease in ATP levels after 30 min of CDME loading in the present study still might be sufficient for normal Na,K-ATPase activity. Ben-Nun et al. (6) showed a decrease in ${ }^{86} \mathrm{Rb}^{+}$influx, which was measured after $10 \mathrm{~h}$ of loading with $1 \mathrm{mM}$ CDME. According to our data, cells are not viable after such a period and cell death might have caused a decrease in ${ }^{86} \mathrm{Rb}^{+}$influx.

A burst of superoxide after CDME loading demonstrated in our study can either be attributed to mitochondrial dysfunction, resulting in increased free radical generation or to decreased capacity of the cell to deal with oxidative stress. Extensive free radical damage probably led to cell death, the rate of which was dependent on the concentration of CDME and the time of exposure, as shown by the viability experi- ments. It is striking that only in the first pioneering paper of Foreman et al. (21), the results of the viability assay are mentioned: using trypan blue exclusion test, they demonstrated that after 10-min exposure to $2 \mathrm{mM} \mathrm{CDME}, 90 \%$ of rat proximal tubules remained viable. These results are in concordance with those obtained in our study. Longer loading times and higher CDME concentrations used by others might have reduced cell viability, which would influence the outcome. Because we could not exclude that PTEC are more resistant to CDME toxicity compared with fibroblasts, we performed the viability assay in human PTEC line HK-2 after exposure to different concentrations of CDME. The results obtained were similar to those obtained in the fibroblasts.

Do the present data mean that the hypothesis of altered ATP metabolism being a major player in the pathogenesis of cystinosis should be rejected? Previous studies show a moderate, but a significant decrease in intracellular ATP content in cultured cystinotic fibroblasts, polymorphonuclear cells, and primary PTEC exposed to hypoxia $(13,15)$.

Remarkably, in the current study, ATP levels of cystinotic fibroblasts were in the range of control values. This discrepancy with our previous results could be attributed to a biologic variability in a small number of observations and an overlap between cystinotic and control values (13). Additionally, swollen mitochondria have been described in kidney tissue of cystinose patients and PTEC of $\mathrm{Ctns}^{-/-}$mice, indicating that ATP metabolism may play a role in cystinosis (22-24). However, mitochondrial ATP generation and Na,K-ATPase activity were normal in cystinotic fibroblasts (13). Furthermore, $\mathrm{Ctns}^{-1-}$ mice model exhibit no signs of proximal tubular dysfunction (24). Because cultured fibroblasts preferentially use anaerobic glycolysis for the ATP production, it cannot be excluded that cystine accumulation causes the alteration of the mitochondrial oxidative phosphorylation in metabolically active cells in vivo $(13,25)$. Therefore, studies in human cystinotic PTEC after the inhibition of the glycolytic pathway and stimulation of the mitochondrial respiration will be of paramount importance for further exploring of the ATP hypothesis. Measurement of ATP content in vivo in cystinotic patients by magnetic resonance spectroscopy might underscore the hypothesis.

In conclusion, CDME loading of control fibroblasts resulted in a toxic effect on the mitochondrial ATP generation. In addition, CDME acted in a dose-dependent manner on the viability of the cells and increased superoxide production. These findings question the validity of CDME loading model for studying the pathogenesis of cystinosis and suggest that the hypothesis of altered mitochondrial respiration obtained in this model should be reconsidered. Further studies in metabolically active PTEC carrying CTNS gene mutations and in vivo measurements of intracellular ATP status in cystinotic patients are required for definitive confirmation or rejection of the hypothesis of ATP alterations being the keystone in the pathogenesis of cystinosis.

\section{REFERENCES}

1. Town M, Jean G, Cherqui S, Attard M, Forestier L, Whitmore SA, Callen DF, Gribouval O, Broyer M, Bates GP, Van’t Hoff W, Antignac C 1998 A novel gene 
encoding an integral membrane protein is mutated in nephropathic cystinosis. Nat Genet 18:319-324

2. Gahl WA, Thoene JG, Schneider JA 2002 Cystinosis. N Engl J Med 347:111-121

3. Gahl WA, Bashan N, Tietze F, Schulman JD 1984 Lysosomal cystine countertransport in heterozygotes for cystinosis. Am J Hum Genet 36:277-282

4. Goldman R, Kaplan A 1973 Rupture of rat-liver lysosomes mediated by L-aminoacid esters. Biochim Biophys Acta 318:205-216

5. Reeves JP 1979 Accumulation of amino-acids by lysosomes incubated with aminoacid methyl-esters. J Biol Chem 254:8914-8921

6. Ben-Nun A, Bashan N, Potashnik R, Cohen-Luria R, Moran A 1992 Cystine dimethyl ester reduces the forces driving sodium-dependent transport in Llc-Pk1 cells. Am J Physiol 263:C516-C520

7. Foreman JW, Benson L 1990 Effect of cystine loading and cystine dimethylester on renal brushborder membrane-transport. Biosci Rep 10:455-459

8. Salmon RF, Baum M 1990 Intracellular cystine loading inhibits transport in the rabbit proximal convoluted tubule. J Clin Invest 85:340-344

9. Ben-Nun A, Bashan N, Potashnik R, Cohen-Luria R, Moran A 1993 Cystine loading induces Fanconi's-syndrome in rats: in vivo and vesicle studies. Am J Physiol 265:F839-F844

10. Coor C, Salmon RF, Quigley R, Marver D, Baum M 1991 Role of adenosinetriphosphate (ATP) and NaK ATPase in the inhibition of proximal tubule transport with intracellular cystine loading. J Clin Invest 87:955-961

11. Foreman JW, Benson LL, Wellons M, Avner ED, Sweeney W, Nissim I, Nissim I 1995 Metabolic studies of rat renal tubule cells loaded with cystine - the cystine dimethylester model of cystinosis. J Am Soc Nephrol 6:269-272

12. Baum M 1998 The Fanconi syndrome of cystinosis: insights into the pathophysiology. Pediatr Nephrol 12:492-497

13. Levtchenko EN, Wilmer MJ, Janssen AJ, Koenderink JB, Visch HJ, Willems PH, Graaf-Hess A, Blom HJ, van den Heuvel LP, Monnens LA 2006 Decreased intracellular ATP content and intact mitochondrial energy generating capacity in human cystinotic fibroblasts. Pediatr Res 59:287-292

14. Wilmer MJ, Graaf-Hess A, Blom HJ, Dijkman HB, Monnens LA, van den Heuvel LP, Levtchenko EN 2005 Elevated oxidized glutathione in cystinotic proximal tubular epithelial cells. Biochem Biophys Res Commun 337:610-614
15. Laube GF, Shah V, Stewart VC, Hargreaves IP, Haq MR, Heales SJ, van't Hoff WG 2006 Glutathione depletion and increased apoptosis rate in human cystinotic proximal tubular cells. Pediatr Nephrol 21:503-509

16. Cetinkaya I, Schlatter E, Hirsch JR, Herter P, Harms E, Kleta R 2002 Inhibition of $\mathrm{Na}+$-dependent transporters in cystine-loaded human renal cells: Electrophysiological studies on the Fanconi syndrome of cystinosis. J Am Soc Nephrol 13:2085-2093

17. Graaf-Hess A, Trijbels F, Blom H 1999 New method for determining cystine in leukocytes and fibroblasts. Clin Chem 45:2224-2228

18. Visch HJ, Rutter GA, Koopman WJ, Koenderink JB, Verkaart S, de Groot T, Varad A, Mitchell KJ, van den Heuvel LP, Smeitink JA, Willems PH 2004 Inhibition of mitochondrial $\mathrm{Na}+-\mathrm{Ca} 2+$ exchange restores agonist-induced ATP production and $\mathrm{Ca} 2+$ handling in human complex I deficiency. J Biol Chem 279:40328-40336

19. Zhao H, Joseph J, Fales HM, Sokoloski EA, Levine RL, Vasquez-Vivar J, Kalyanaraman B 2005 Detection and characterization of the product of hydroethidine and intracellular superoxide by HPLC and limitations of fluorescence. Proc Natl Acad Sci USA 102:5727-5732

20. Yang Y, Balcarcel RR 2004 96-well plate assay for sublethal metabolic activity. Assay Drug Dev Technol 2:353-361

21. Foreman JW, Bowring MA, Lee J, States B, Segal S 1987 Effect of cystine dimethylester on renal solute handling and isolated renal tubule transport in the rat a new model of the Fanconi syndrome. Metabolism 36:1185-1191

22. Jackson JD, Smith FG, Litman NN, Yuile CL, Latta H 1962 The Fanconi syndrome with cystinossis. Electron microscopy of renal biopsy specimens from five patients. Am J Med 33:893-910

23. Spear GS, Slusser RJ, Tousimis AJ, Taylor CG, Schulman JD 1971 Cystinosis. An ultrastructural and electron-probe study of the kidney with unusual findings. Arch Pathol 91:206-22

24. Cherqui S, Sevin C, Hamard G, Kalatzis V, Sich M, Pequignot MO, Gogat K, Abitbol M, Broyer M, Gubler MC, Antignac C 2002 Intralysosomal cystine accumulation in mice lacking cystinosin, the protein defective in cystinosis. Mol Cell Biol 22:7622-7632

25. Robinson BH 1996 Use of fibroblast and lymphoblast cultures for detection of respiratory chain defects. Methods Enzymol 264:454-464 